\title{
A case report of giant chondroma arising at pharynx nasalis
}

\section{Jun Feng ${ }^{1}$, Jiumei Yang ${ }^{1}$, Li Li ${ }^{2 *}$, Tao Peng ${ }^{1}$ and Yuanzhuo Zhuang ${ }^{1}$}

${ }^{1}$ Department of Otolaryngology-Head and Neck Surgery, Nanchong Central Hospital, Second Clinical Medical College of North Sichuan Medical College, Nanchong 637000, China

${ }^{2}$ Department of Pathology, Affiliated Hospital of North Sichuan Medical College, Nanchong 637000, China

\begin{abstract}
Chondromas are common slow-growing benign tumors arising at many anatomic sites, but the tumor in situ of pharynx nasalis is very rare without exact etiology. The clinical manifestation about the disease mainly includes the tumorous pressure and autochthonous bleeding. A twelve-year-old girl was seen for the complaint of progressive nasal obstruction and snoring over one year, with signs of hearing degression in the right ear for one month. We carried out this operation by sinus nasalis endoscope and electrodrill transnasal approach. The final diagnosis of chondromas is confirmed by pathology. For five years's follow-up, the patient was asymptomatic without evidence of recurrence.
\end{abstract}

\section{Introduction}

Chondromas are benign tumors that may be found in many parts of body, but it is rare that it develops at the region of nasal parts, especially at pharynx nasalis. Furthermore, the neoplasm at pharynx nasalis is hard to excise completely so that recurrence may ocurr. Now here we report a case of a giant chondroma in the particular location.

\section{Case report}

A twelve-year-old girl was seen for the complaint of progressive nasal obstruction and snoring over one year, with signs of hearing degression in the right ear for one month. The electron pharyngorhinoscopy showed there was a smooth-surfaced hard neoplasm on the posterior wall of nasopharynx, which measured $4.0 \times 5.0 \mathrm{~cm}$. Her right ostium pharyngeum tubae auditoriae couldn't be seen very well because of the tumorous pressure and ancipitous choana nariums were almost obstructed. The CT (Figures 1A-1C) presented that there was an anormal soft tissue in the right pharynx nasalis and pars oralis pharynges, measuring $3.0 \times 3.5 \times 5.0 \mathrm{~cm}$, with clear bouncary and irregularly granular calcifications. There was no abnormality seen in all the other examinations.

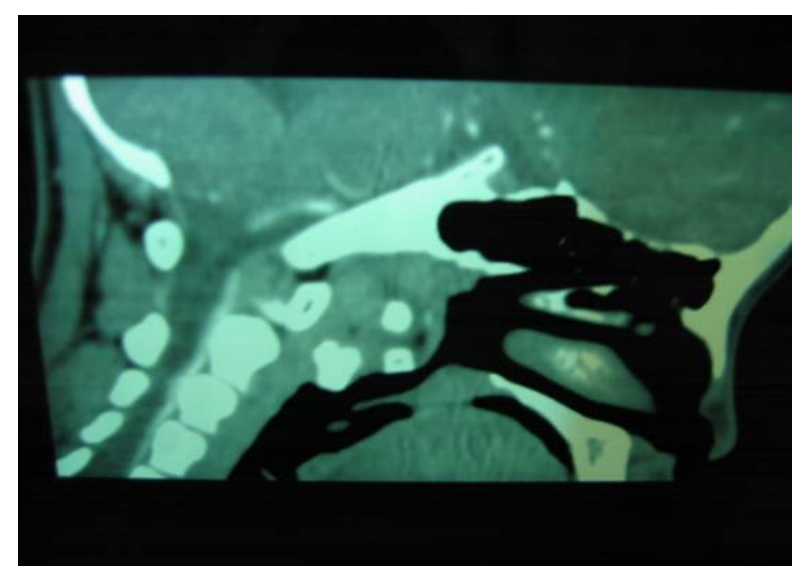

Figure 1A. CT at anteroposterior axes position

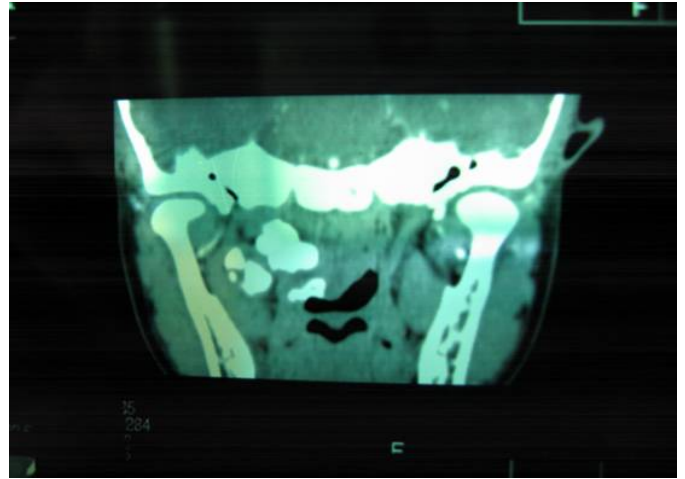

Figure 1B. CT at coronal position

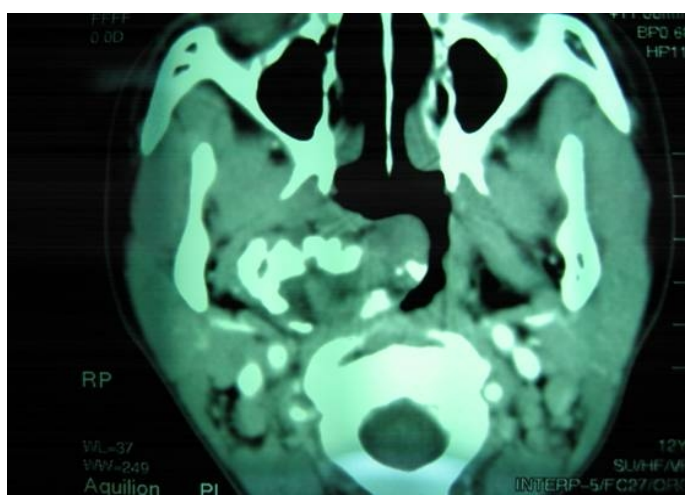

Figure 1C. $\mathrm{CT}$ at axial position

It shows the location and imaging characteristic of the tumor from three differient angles. No bony destruction can be seen (Figures 1A-1C).

Correspondence to: Li Li, Department of Pathology, Affiliated Hospital of North Sichuan Medical College, Nanchong 637000, China, Tel: 15892772916; E-mail: 15984876269@163.com

Received: October 24, 2017; Accepted: November 25, 2017; Published: November 28, 2017 
Under general anaesthesia in controlling low blood pressure, the operation was performed transnasal approach with sinus nasalis endoscope and electrodrill which made in Stryker and Medtronic corporation respectively. We resected the Chondroma as following step: (1) Shrinking mycteric and nasopharyngeal mucosae and nasal concha with cotton slice soddened normal saline (NS) containing adnephrin $4 \mathrm{mg}$, summation 3 times about every 5 minutes one time, (2) under sinus nasalis endoscope, sectioning mucosae covering on surface of Chondroma about $1.5 \mathrm{~cm}$, exposing and grinding Chondroma and (3) using absorbable hemostatic gauze to fill into wound surface to hematischesis. We found the neoplasm composed of three independent smooth pieces of milky hard tumor tissues. The biggest piece measured up to $5 \times 4 \times 3 \mathrm{~cm}$ and neither obvious adherence nor destruction of bone occurred. Pathological examination revealed well-differentiated hyaline cartilage cells without pathologic caryocinesia arranged in an irregularly lobular pattern. The basilaris substantia with some scutellate calcareous infarcts was sparse and took on cystose somewhere. Pathological conclusion was a chondroma. The patient was nasal obstruction and snoring free, whose acoustic sensibility was elevated by fifteen decibels after one week. Her hearing was normal one month later. For five years follow-up, the patient was asymptomatic without evidence of recurrence.

\section{Discussion}

The exact etiology of chondromas is unknown. Most authors agree that it may originate from heterotopic embryonal cartilaginous cell or the remain of skul cartilage [1]. Kilby et al. [2] in 1977 divided the regions of chondromas in nasal cavity and accessory nasal cavity into six districts (1) ethmoidal sinus and nasal cavity except median septum 50\% (2) median septum 17\% (3) maxillary bone and antrum maxillae 18\% (4) hard palate 6\% (5) pharynx nasalis, sphenoid cell and auditory tube $6 \%(6)$ nasal alar cartilage $3 \%$. The clinical manifestation mainly includes the tumorous pressure and autochthonous bleeding. But the volume of bleeding is modicus and sometimes it only gives an appearance of blood in nasal mucus and aspirated bloody sputum. The pressure may lead to nasal obstruction, headache, hearing degression or encroachment on proximal tissues. Jiangming Zhao et al. [3] state that in earlier period CT can reveal a benign tumor, but in later period it presents malignant tumor's manifestation. In JingWei Shang's [4] opinion, CT far outweighs MRI in the diagnosis of basalis cartilaginous tumor, respecting that $\mathrm{CT}$ is better than MRI in the discrimination for the typical ossification and calcification. Therefore CT can play great importance in preliminary confirmming the qualitation of the neoplasm. But in revealing the neoplasm's location and adjacency to proximal tissues, MRI is superior to CT. Murthy et al. [5] consider that complete surgical removal is the best treatment of choice. Recurring possibility will not be diminished by surgery combining radiotherapy together or merely radiotherapy and malignant degeneration into chondrosarcoma may occur after partial resection. So process tissues together with proximal periosteum or perichondrium should be cut, whose cutting edge is located on the spot at a distance of 0.5 to 1.0 $\mathrm{cm}$ from the neoplasm bouncary. Notwithstanding a benign neoplasm, it is considerably destructive, as a result of incomplete excision and rapid growth of the neoplasm, which will result in encroaching on proximal tissues and extending to erode other places such as fossa orbitalis, intracalvarium. Accordingly, it is clinically treated as a kind of malignant or semi-malignant tumor and long-term follow-up is also needed. In this case, we accomplished this operation thoroughly using sinus nasalis endoscope and electrodrill in a small incisal opening, and followed-up five years without recurrence.

Chondromas are benign tumors that rarely arise at pharynx nasalis. Considering their propensity to malignant transformation into chondrosarcoma after partial resection of tumor, every attempt should be made to total removal of the tumor, which makes the longterm prognosis good. Cure is usual with marginal and intralesional excision under a proper approach. It is obvious that there are a lot of preponderance such as a wide campus visualis, small incisal opening, excision thoroughly, little hemorrhage, no recurrence to ues sinus nasalis endoscope and electrodrill transnasal approach to finish this operation.

\section{Funding}

This work was supported by grants from the Education Department of Sichuan Province (CN) (13ZB0241) the Doctoral Scientific Research Foundation of North Sichuan Medical College (CBY13-QD-08) and Bureau of science \& Technology Intellectual Property Nanchong City (16YFZJ0021).

\section{Acknowledgement}

It is certain that the IRB of Nanchong Central Hospital, Second Clinical Medical College of North Sichuan Medical College has agreed this Case Report of Giant Chondroma Arising at Pharynx nasalis for publication.

\section{References}

1. Kretzschmar HA, Eggert HR, Beck U, Furmaier R (1989) Intracranial chondroma: case report. Surg Neurol 32:121-5.

2. Kilby D, Ambegaokar AG (1977) The nasal chondroma 2 case reports and a survey of the literature. J Laryngol Otol 91: 415-26. [Crossref]

3. Jiang-Ming Z, Jing-Wei S, Jian-Ping D, et al. (2002) Chondroma of skull base: CT and MR imaging. J Chin Clin Med Imaging 13: 200-203.

4. Jing-Wei S, Jian-Ping D, Pei-Yi G, et al. (2001) Imaging diagnosis of skull base chondromas. J Chin Prac Radiation 17: 343-345.

5. Murthy DP, Gupta AC, Sengupta SK, Dutta TK, Pulotu ML (1991) Nasal Cartilaginous tumour. J Laryngol Otol 105-670. [Crossref]

Copyright: (C2017 Feng J. This is an open-access article distributed under the terms of the Creative Commons Attribution License, which permits unrestricted use, distribution, and reproduction in any medium, provided the original author and source are credited. 